\section{Disminución en la frecuencia de infección por Helicobacter pylori en endoscopías digestivas altas}

\author{
DANIEL DA COSTA ${ }^{1}$, FRANCESCA GUIDOTTI ${ }^{2}$, \\ NICOLAS CABELLO ${ }^{a}$, FRANCISCO TRIGO $^{a}$, CATERINA CONTRERAS $^{a}$, \\ FRANCISCA VERGARA ${ }^{\mathrm{a}}$, JUAN PABLO MIRANDA ${ }^{3, \mathrm{~b}}$, \\ CRISTIÁN MONTENEGRO ${ }^{1}$, PABLO MUÑOZ1 ${ }^{1}$, ZOLTÁN BERGER $^{1}$
}

\section{Helicobacter pylori infection detected during upper gastrointestinal endoscopy}

Background: Helicobacter pylori (HP) is the most widespread chronic human infection worldwide and the most important pathogenic factor of gastric cancer. The calculated prevalence at the Clinical Hospital of the University of Chile from 2002 to 2005 was 44.9\%. Aim: To determine the current prevalence of HP in patients undergoing an upper gastrointestinal endoscopy (UGI) and analyze its distribution according to age and endoscopic findings. Material and Methods: We reviewed 3.433 UGI performed during the year 2015, selecting those in which rapid urease test (RUT) was done. A positive $R U T$ or a positive gastric biopsy (GB) were considered as HP infection. Results: RUT was done in 1862 UGI (55\%) performed in patients aged $51 \pm 17$ years, (66\% women). In 23\% of these endoscopies, the RUT was positive. A GB was obtained 43\% of endoscopies and 30\% were positive for HP. In 105 patients the RUT was negative and the GB positive (rendering a 19.5\% false negative rate). HP was detected by RUT and GB in $29 \%$ of endoscopies. The highest prevalence of infection (38.1\%) was found between 40 and 49 years. $H P$ infection had odds ratio of 4.24 for nodular gastropathy, 2.63 for gastric ulcer and 2.14 for duodenal ulcer $(p<0.05)$. Conclusions: HP prevalence in our center decreased significantly from $44.9 \%$ to $28.9 \%$ in 11 years. False negative RUT results may bias this finding. The use of proton pump inhibitors and antimicrobials that can interfere with the detection of HP should be registered to properly analyze the results of the RUT.

(Rev Med Chile 2018; 146: 555-561)

Key words: Endoscopy, Gastrointestinal; Helicobacter pylori; Prevalence; Upper Gastrointestinal Tract; Urease.
'Departamento de Medicina, Sección de Gastroenterología, Hospital Clínico Universidad de Chile. Santiago, Chile.

${ }^{2}$ Departamento de Medicina, Sección de Medicina Interna, Hospital Clínico Universidad de Chile. Santiago, Chile.

${ }^{3}$ Departamento de Medicina, Unidad de Paciente Crítico Hospital Clínico Universidad de Chile. Santiago, Chile.

anterno de Medicina, Universidad de Chile.

${ }^{\mathrm{b}}$ Magíster en Estadística.

Trabajo no recibió financiamiento Los autores declaran no tener conflictos de interés.

Recibido el 4 de junio de 2017 , aceptado el 24 de abril de 2018.

Correspondencia a:

Zoltán Berger Fleiszig.

Departamento de Medicina, Sección de Gastroenterología, Hospital Clínico Universidad de Chile.

Santos Dumont 999,

Independencia. Santiago, Chile.

Fono: 29788350.

berger.zoltan@gmail.com

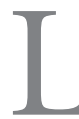

a infección por Helicobacter pylori (HP) es la infección crónica más extendida a nivel mundial. Afecta a $50 \%$ de la población, llegando a $90 \%$ en países subdesarrollados, mientras que en otros países, como Australia, puede llegar a afectar sólo a $20 \%{ }^{1}$. En Chile, mediante estudio serológico en la Encuesta Nacional de Salud del año 2007, se estableció una prevalencia de $73 \%$, que es la más citada a nivel nacional ${ }^{2}$. En población de La Florida, a través de test de ureasa, se encontró una prevalencia de $78 \%{ }^{3}$. En contraste, se encontró una prevalencia de $44,9 \%{ }^{4}$ 
en un estudio realizado en el Hospital Clínico de la Universidad de Chile (HCUCh) durante los años 2002 a 2005, con test de ureasa rápido(TUR) fabricado en nuestro laboratorio, que es el mismo utilizado actualmente (Tabla 1). Estas diferencias de prevalencia pueden estar relacionadas con el nivel socioeconómico, condiciones de higiene, la edad y la etnia.

HP es el factor etiopatogénico más importante de cáncer gástrico ${ }^{2,8-12}$, el cual en Chile es la primera causa de muerte por cáncer en hombres y la tercera en mujeres ${ }^{13,14}$. Hoy en día, el mecanismo oncogénico es entendido por la colonización de HP sobre el epitelio gástrico, produciendo una respuesta inflamatoria local y sistémica, lo que conduce a la conocida cascada de gastritis aguda, gastritis crónica, gastritis crónica atrófica, metaplasia intestinal y displasia, considerándose éstas tres últimas como lesiones premalignas ${ }^{15}$. Estudios recientes han logrado demostrar reducción en la incidencia de cáncer gástrico mediante la erradicación de HP a nivel poblacional, que tiene mayor efecto en zonas de mayor incidencia de cáncer gástrico ${ }^{16-19}$. Por otro lado, HP se ha relacionado con úlcera péptica gastroduodenal, linfoma gástrico, dispepsia, halitosis ${ }^{20}$, hiperémesis gravídica ${ }^{6}$, anemia ferropénica idiopática y púrpura trombocitopénico idiopático.

El objetivo del trabajo es estimar la prevalencia de infección por HP en pacientes derivados a endoscopía digestiva alta (EDA) en el HCUCh y correlacionarla con la indicación de la EDA, la edad y los hallazgos endoscópicos. Además, al comparar los resultados con los publicados anteriormente por nuestro grupo, buscar eventuales cambios en la frecuencia de infección por HP y de las enfermedades relacionadas con la bacteria.

\section{Material y Método}

Estudio transversal mediante revisión retrospectiva de 3.433 pacientes derivados a EDA desde enero a diciembre de 2015. Se seleccionaron todas aquellas con TUR. Se excluyeron los pacientes a los que no se les realizó TUR y a aquellos en que no se encontró la información completa, tanto del resultado del TUR como de la histología.

Se utilizaron endoscopios Olympus GIF-H180 y Fujinon de la serie 500, con técnica estándar luego de consentimiento informado. Los procedimientos fueron realizados por endoscopistas del HCUCh.

Se utilizó TUR, el cual consiste en un 1 cc de solución de urea $10 \%$ p/v y rojo fenol contenida en tubo Eppendorf, la cual utiliza el rojo fenol como indicador de $\mathrm{pH}$ para detectar la hidrólisis de la urea cuando está presente HP, considerándose el resultado como positivo cuando el color de la solución vira de color amarillo a magenta dentro de algunos minutos a dos horas. Esta solución es fabricada en el Laboratorio de Gastroenterología del HCUCh, con reportes de sensibilidad de $83 \%$, $96,6 \%$ y $98,2 \%$ y especificidad de $86 \%, 99,2 \%$ y $99 \%$, en las distintas series ${ }^{21-23}$, que no es inferior a los valores obtenidos con los tests comercializados. En un estudio previo, el TUR fabricado en HCUCh fue comparado con cultivo de HP, demostrando 1 falso negativo de 50 cultivos positivos ${ }^{4}$. Según recomendación local, se tomó una muestra de antro y otra de cuerpo para TUR. Las biopsias gástricas fueron siempre múltiples, tomadas según protocolo Sydney y desde las lesiones, las que fueron examinadas con tinción de Giemsa. Se consideró infección por HP cuando el TUR fue positivo $y / 0$ hubo presencia de HP en al menos una biopsia

Tabla 1. Prevalencia de infección por Helicobacter pylori publicada en los últimos 15 años

\begin{tabular}{|cllcc|}
\hline Año (referencia) & Población & $\begin{array}{c}\text { Técnica de } \\
\text { medición }\end{array}$ & $\begin{array}{c}\text { n de pacientes } \\
\text { Pospital de Temuco }\end{array}$ & $\begin{array}{c}\text { Prevalencia } \\
\text { de infección }\end{array}$ \\
\hline $2000^{5}$ & Histología & 200 & $82 \%$ \\
\hline $2007^{4}$ & Endoscopía HCUCh & Test de ureasa & 276 & $45 \%$ \\
\hline $2017^{3}$ & Encuesta Nacional de Salud & Serología lgG & 2.615 & $73 \%$ \\
\hline $2014^{6}$ & Endoscopía La Florida & Test de ureasa & 5.644 & $78 \%$ \\
$2016^{7}$ & Endoseres embarazadas Concepción & Serología lgG & 274 & $69 \%$ \\
\hline
\end{tabular}

HCUCh: Hospital Clínico Universidad de Chile. HCUC: Hospital Clínico Universidad Católica. 
gástrica. Se consideraron los siguientes hallazgos endoscópicos: gastropatía nodular, úlcera gástrica, úlcera duodenal, duodenopatía erosiva, gastropatía erosiva, gastropatía varioliforme, esofagitis y pólipos gástricos. No se obtuvo el antecedente de consumo de antagonistas del receptor $\mathrm{H}_{2}$, inhibidores de la bomba de protones ni uso concomitante de antibióticos.

El trabajo cuenta con la Aprobación del Comité de Ética Científico del HCUCh.

\section{Análisis estadístico}

Se utilizó test de $\chi^{2}$ para establecer diferencias entre la frecuencia de infección por HP y el hallazgo endoscópico, mientras que Odds Ratio (OR) con intervalo de confianza de $95 \%$, corregidos por regresión logística binaria por edad y sexo, para estimar el riesgo de los distintos hallazgos endoscópicos según infección por HP. Se utilizó el programa Stata 12, considerándose significativo un valor $\mathrm{p}<0,05$.

\section{Resultados}

De los 3.433 pacientes sometidos a EDA durante el 2015, se les realizó TUR a 1.901 pacientes, correspondiente a 55\% de las EDA-s. No se tomó TUR en el restante $45 \%$ de las EDA-s. El criterio de realizar o no TUR fue la indicación del médico tratante o los hallazgos endoscópicos evaluados por el endoscopista. Se excluyeron 39 pacientes, cuando el resultado de TUR no fue disponible o el patólogo no informó sobre presencia de HP. Se analizaron los datos de 1.862 pacientes.

La edad promedio fue de $51 \pm 17$ años, rango 16 a 95 años. El 66\% fue de sexo femenino.

El principal motivo de derivación de los pacientes a EDA fue la dispepsia $(35,2 \%)$, seguido por el reflujo gastroesofágico $(17,7 \%)$ (Tabla 2$)$. $\mathrm{HP}$ fue encontrado con significativamente mayor frecuencia en aquellos pacientes cuya indicación fue chequeo previo a cirugía bariátrica (OR 2,06; IC 95\%: 1,33-3,19) y dispepsia (OR 1,33; IC 95\%: $1,08-1,63)$, mientras con significativamente menor frecuencia en pacientes con indicación de EDA por cirrosis (OR: 0,44; IC 95\%: 0,23-0,84). Es decir, la probabilidad de los cirróticos de tener infección por HP fue 2,27 veces menor que de los pacientes sin cirrosis.

El TUR fue positivo en 434 de $1.862(23,3 \%)$. Se tomó también biopsia gástrica en 797 de ellos $(42,8 \%)$. De las biopsias gástricas, $233(29,2 \%)$

Tabla 2. Motivo de realización de la endoscopía digestiva alta y su relación con la frecuencia de infección por Helicobacter pylori

\begin{tabular}{|lccccc|}
\hline $\begin{array}{l}\text { Motivo de realización de } \\
\text { la endoscopía }\end{array}$ & $\mathbf{n}$ & $\mathbf{\%}$ & $\begin{array}{c}\text { Infección } \\
\text { por HP }\end{array}$ & OR* & IC (95\%) \\
\hline Dispepsia & 656 & $35,2 \%$ & $32,8 \%$ & 1,33 & $1,08-1,63$ \\
\hline Reflujo gastroesofágico & 330 & $17,7 \%$ & $29,7 \%$ & 1,04 & $0,81-1,36$ \\
\hline Sin diagnóstico & 174 & $9,3 \%$ & $23,6 \%$ & 0,74 & $0,51-1,06$ \\
\hline Otras & 170 & $9,1 \%$ & $28,8 \%$ & 0,99 & $0,70-1,41$ \\
\hline Control & 149 & $8,0 \%$ & $19,5 \%$ & 0,57 & $0,38-0,87$ \\
Previo cirugía bariática & 85 & $4,6 \%$ & $44,7 \%$ & 2,06 & $1,33-3,19$ \\
\hline Cirrosis & 71 & $3,8 \%$ & $15,5 \%$ & 0,44 & $0,23-0,84$ \\
\hline Hemorragia digestiva & 53 & $2,8 \%$ & $30,2 \%$ & 1,06 & $0,59-1,92$ \\
\hline Anemia en estudio & 47 & $2,5 \%$ & $23,4 \%$ & 0,75 & $0,38-1,47$ \\
\hline Disfagia & 41 & $2,2 \%$ & $26,8 \%$ & 0,89 & $0,45-1,80$ \\
\hline Tamizaje cáncer gástrico & 34 & $1,8 \%$ & $17,6 \%$ & 0,52 & $0,21-1,26$ \\
\hline Baja de peso & 26 & $1,4 \%$ & $26,9 \%$ & 0,9 & $0,38-2,17$ \\
\hline Total & 1.862 & $100,0 \%$ & $28,9 \%$ & & \\
\hline
\end{tabular}

El motivo de realización de la endoscopía fue descrito tal cual estaba anotado en el informe de la endoscopía, esto incluye $9,3 \%$ de endoscopías sin diagnóstico en la orden médica y $8 \%$ de endoscopías por control no especificados en el informe. *Odds Ratio crudos. 
fueron según protocolo de Sydney, en los otros casos se tomaron muestras de las lesiones focales 0 difusas. La biopsia gástrica mostró presencia de HP en 30\%. Hubo 105 pacientes con TUR negativo y biopsia gástrica positiva (19,5\% de falsos negativos de TUR), mientras que en 13 pacientes TUR fue positivo y biopsia gástrica negativa (5\% de falsos negativos de biopsias). Considerando la infección por HP como TUR positivo y/o biopsia gástrica positiva, se encontró una prevalencia de infección de $28,9 \%$ en los 1.862 pacientes estudiados.

La mayor prevalencia de infección se encontró durante la quinta década de la vida, entre los 40 a 49 años de edad, llegando a ser de $38,1 \%$, la cual fue disminuyendo por cada década (Figura 1).

Al relacionar el hallazgo endoscópico con la frecuencia de infección por HP, se encontró que la gastropatía nodular, la úlcera gástrica, la úlcera duodenal, la duodenopatía erosiva y la gastropatía erosiva tuvieron una relación positiva significativa $(\mathrm{p}<0,05)$. El hallazgo endoscópico que tuvo mayor relación con la infección por HP fue la gastropatía nodular (OR 4,24; IC 95\%: 2,9-6,1). Se encontró que la gastropatía varioliforme y la esofagitis no tuvieron relación significativa con la infección por HP, mientras que la presencia de pólipos gástricos se asoció a menor frecuencia de infección por HP (OR 0,51; IC 95\%: 0,4-0,7) (Tabla 3).

Hubo un total de 207 (11,1\%) EDA-s normales. Estas tuvieron infección por HP en $23,2 \%$, lo que implica que los pacientes con EDA normal tienen una tendencia no significativa a no estar infectados por HP (OR 0,72; IC 0,5-1,01, p = 0,06).

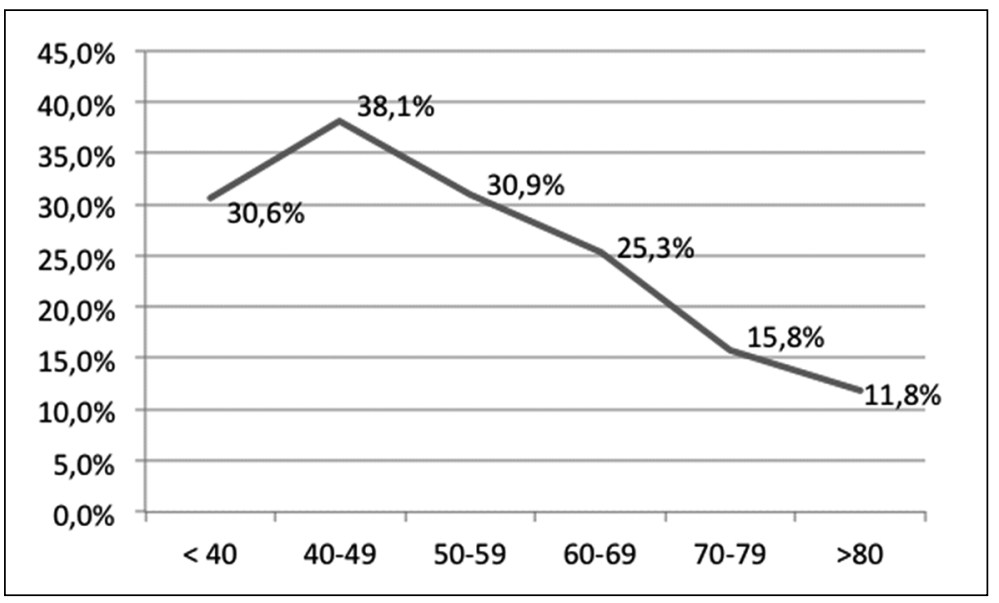

Figura 1. Prevalencia de infección por Helicobacter pylori según década de la vida. La prevalencia de infección por HP por test de ureasa rápido y biopsia gástrica fue de $28,9 \%$. En menores de 40 años fue de $30,6 \%, 40$ a 49 años fue de $38,1 \%, 50$ a 59 años fue de $30,9 \%, 60$ a 69 años fue de 25,3\%, 70 a 79 años fue de $15,8 \%$ y mayores de 80 años fue de $11,8 \%$.

Tabla 3. Relación entre la frecuencia de infección por Helicobacter pylori y el hallazgo endoscópico

\begin{tabular}{|lccccc|}
\hline Hallazgo endoscópico & Frecuencia (\%) & HP (\%) & OR $^{*}$ & IC (95\%) & p** $^{\text {(\%) }}$ \\
\hline Gastropatía nodular & 6,9 & 54 & 4,24 & $2,9-6,1$ & 0,0001 \\
\hline Úlcera gástrica & 3,7 & 51 & 2,63 & $1,6-4,2$ & 0,0001 \\
\hline Úlcera duodenal & 2,7 & 46 & 2,14 & $1,2-3,8$ & 0,008 \\
\hline Duodenopatía erosiva & 28,0 & 37 & 1,86 & $1,5-2,3$ & 0,0001 \\
\hline Gastropatía erosiva & 51,5 & 32 & 1,36 & $1,1-1,7$ & 0,0024 \\
Gastropatía varioliforme & 5,7 & 31 & 1,12 & $0,7-1,7$ & 0,61 \\
\hline Esofagitis & 22,6 & 31 & 1,1 & $0,9-1,4$ & 0,3 \\
\hline Normal & 11,1 & 23 & 0,72 & $0,5-1,01$ & 0,06 \\
\hline Pólipos gástricos & 12,8 & 18 & 0,51 & $0,4-0,7$ & 0,0001 \\
\hline
\end{tabular}

${ }^{*}$ Corregidos por Regresión Logística Binaria. ${ }^{* *} \chi^{2}$. 


\section{Discusión}

Desde que Marshall y Warren describieron la infección por HP en 1983 y su asociación con patología gastroduodenal, ha existido una intensa investigación que ha llevado actualmente a la recomendación de erradicación de HP a toda persona infectada ${ }^{24}$ para la prevención primaria del cáncer gástrico en comunidades con alto riesgo (Maastricht $\mathrm{V})^{25}$. Esto ha incrementado la importancia de la búsqueda de HP en la mayoría de nuestros pacientes, ya que el tratamiento relativamente precoz de la infección, antes de producir un daño irreversible, está considerado como prevención primaria del cáncer gástrico particularmente en comunidades de alto riesgo, como Chile.

Nuestro trabajo mostró una reducción significativa $(\mathrm{p}<0,001)$ en la frecuencia de infección por HP en el HCUCh de 44,9\% (2002 a 2005) a $28,9 \%$ (2016). Esta reducción de $16 \%$ en 11 años puede deberse a varios factores, como la indicación universal de erradicación de HP a todo paciente infectado, tratamiento recibido eventualmente antes de la EDA y a mejoría del nivel socioeconómico e higiénico de la población.

Hay limitaciones en nuestro hallazgo de baja prevalencia de infección por HP, principalmente no haber considerado el tratamiento con inhibidores de la bomba de protones. A pesar de la recomendación de suspenderlos dos semanas previo a exámenes diagnósticos de infección por $\mathrm{HP}^{25}$, ha sido reportado su uso en otros centros de $47 \%{ }^{26}$ y $70 \%{ }^{27}$ en pacientes derivados a EDA, lo que disminuye la sensibilidad del TUR y de la biopsia gástrica hasta $70 \%$ (30\% de falsos negativos) para diagnóstico de infección por $\mathrm{HP}^{28}$. Tampoco consideramos otros factores que disminuyen la sensibilidad del TUR al reducir la carga bacteriana de HP, como la gastritis crónica atrófica, la metaplasia intestinal, la hemorragia digestiva y el uso reciente de antibióticos, ya que concentraciones bacterianas menores de $10^{4}$ pueden resultar en falsos negativos ${ }^{29}$. Estos factores pueden explicar nuestros hallazgos, donde hubo 105 pacientes con TUR negativo y biopsia gástrica positiva para infección por HP, que corresponde a $19,5 \%$ de falsos negativos. Otros factores fueron no tener información de eventual erradicación previa de HP y la recolección retrospectiva de los datos. La toma selectiva de muestra puede interferir también con los resultados, pero en la publicación previa se analizaron datos obtenidos con similares criterios que permite comparar los resultados obtenidos en los dos períodos.

Se tomó muestra para TUR ureasa en sólo 55\% de las EDA-s. Esto es explicado por la tendencia a relacionar la infección de HP sólo con hallazgos endoscópicos patológicos, mientras que la realización de TUR en pacientes asintomáticos con EDA normal con el fin de prevención primaria del cáncer gástrico aún no forma parte de la rutina cotidiana. Creemos que a medida que se acepte ampliamente el rol de la infección por HP en la etiopatogenia y prevención primaria del cáncer gástrico, se difundirá la toma de TUR a mayor número de pacientes, incluidos principalmente jóvenes asintomáticos con EDA normal.

Nuestro trabajo confirmó la asociación entre la infección por HP y la gastropatía nodular, la úlcera gástrica, la úlcera duodenal, la duodenopatía erosiva y la gastropatía erosiva, siendo la asociación más fuerte con gastropatía nodular (54\% de infección, OR 4,24; IC 95\%: 2,9-6,1, p = 0,0001). Se encontró que la presencia de pólipos gástricos se asoció a menor frecuencia de infección por HP $(12,8 \%$ de infección, OR 0,51; IC 95\%: 0,4-0,7, p = 0,0001). Esto puede deberse a que el tratamiento con inhibidores de bomba de protones aumenta la frecuencia de pólipos gástricos y reduce la sensibilidad del TUR y biopsia gástrica, ya que esta asociación no ha sido reportada.

La infección por HP se encontró 2,27 veces menos en cirróticos. Esto puede ser explicado tanto por la menor sensibilidad del $\operatorname{TUR}^{30}$ y la menor prevalencia de HP en estos pacientes ${ }^{31}$.

Los estudios realizados en los años 1980s y 1990s mostraron que la prevalencia de úlcera péptica en pacientes derivados a EDA era de $15 \%{ }^{32}$, las que estaban asociadas a infección por HP en $90 \%$ de úlcera duodenal y en $70 \%$ de úlcera gástrica $^{33}$. La úlcera péptica posteriormente ha ido disminuyendo en prevalencia y asociando cada vez menos a HP, sobrepasando la úlcera gástrica a la úlcera duodenal ${ }^{34}$. Esto fue confirmado también en nuestra serie: la úlcera gástrica $(3,7 \%)$ fue más frecuente que la úlcera duodenal $(2,7 \%)$, y sólo $51 \%$ de las úlceras gástricas y $46 \%$ de las úlceras duodenales fueron positivas para infección por HP, lo que puede deberse al mayor uso de antiinflamatorios no esteroidales. La baja frecuencia de úlcera péptica en nuestro centro es concordante con nuestra baja prevalencia de infección por HP 
$(28,9 \%)$, lo que coincide con lo encontrado en otras series ${ }^{26}$. En contraste, en La Florida, Chile, con una prevalencia de infección por HP de 78\%, se encontró mayor prevalencia de úlcera péptica, con $8,1 \%$ de pacientes con úlcera gástrica y $6,4 \%$ de pacientes con úlcera duodenal ${ }^{3}$. Estos hallazgos apoyan la asociación de HP con úlcera péptica gastroduodenal.

No tenemos datos fidedignos de la frecuencia de hallazgo de cáncer gástrico en estos dos períodos en nuestro centro, sin embargo, según la estadística nacional disponible hubo una disminución de mortalidad por cáncer gástrico en Santiago de 16,9 por 100.000 habitantes en el año 2000 a 12,9 por 100.000 habitantes en el año $2011^{13}$, lo que puede estar relacionado a la disminución en la prevalencia de infección por HP.

\section{Conclusión}

Se buscó infección por HP en 55\% de las EDA-s realizadas en 2015 y su prevalencia fue $28,9 \%$, demostrando una reducción significativa en nuestro centro de $16 \%$ en 11 años. También se observó una baja frecuencia de úlcera péptica duodenal y gástrica, probablemente relacionada a esta reducción de la infección por HP. Los resultados falsos negativos (19,5\%) del TUR, en parte pueden ser resultado del uso no registrado de inhibidores de bomba de protones autoprescritos. Por consiguiente, recomendamos registrar el uso de inhibidores de la bomba de protones y de antibióticos en la orden y en el informe de la EDA. En el caso de uso de éstos, aparte de muestra para TUR, sugerimos también tomar biopsias gástricas, para aumentar el rendimiento diagnóstico de infección por HP. Por la importancia demostrada de erradicación de HP en la prevención primaria de cáncer gástrico, recomendamos la toma de muestra para TUR en todos los pacientes menores de 50 años, aún con examen endoscópico normal. Además, sugerimos controlar la erradicación de la infección por HP durante los controles endoscópicos necesarios por la patología encontrada y aún en los casos sin necesidad de control endoscópico, con test no invasivo, ya sea test de aire espirado con urea marcada o, en primer lugar, el antígeno de HP en deposiciones. Este simple y fácilmente accesible test podría servir también para estudio poblacional.
Agradecimientos: A Mauricio Venegas y Carmen Hurtado por discusión y comentarios.

\section{Referencias}

1. Latorre R, Gallo G. Helicobacter pylori: su importancia práctica en la gastroenterología. Revista Médica Clínica Las Condes 2008; 19: 23-9.

2. Ferreccio C, Rollán A, Harris PR, Serrano C, Gederlini A, Margozzini P, et al. Gastric Cancer is Related to Early Helicobacter pylori Infection in a High-Prevalence Country. Cancer Epidemiol Biomarkers Prev 2007; 16 (4): 662-7.

3. Ortega JP, Espino A, Calvo BA, Verdugo P, Pruyas M, Nilsen E, et al. Infección por Helicobacter pylori en pacientes sintomáticos con patología gastroduodenal benigna: Análisis de 5.664 pacientes. Rev Med Chile 2010; 138 (5): 529-35.

4. Toledo H, Defilippi C, Madrid AM, Defilippi C, Vallejos C, Cáceres D, et al. Prevalencia de la infección por $\mathrm{He}$ licobacter pylori según ensayo de la ureasa en pacientes derivados a la Unidad de Endoscopía del Hospital Clínico de la Universidad de Chile. Rev Hosp Cli Uni Chi 2007; 18: 189-93.

5. Araya JC, Villaseca MA, Roa I, Roa JC. Helicobacter pylo$r i$ and chronic gastritis: relationship between infection and inflammatory activity in a high risk population for gastric cancer. Rev Med Chile 2000; 128 (3): 259-65.

6. Poveda GF, Carrillo KS, Monje ME, Cruz CA, Cancino AG. Helicobacter pylori infection and gastrointestinal symptoms on Chilean pregnant women. Rev Assoc Med Bras 2014; 60 (4): 306-10.

7. Latorre G, Jiménez M, Robles C, Jensen E, Ramos B, Fritzsche M, et al. Aumento de Detección de Lesiones Gástricas Premalignas mediante Protocolo de Sydney en comparación con biopsias no protocolizadas. Gastroenterol latinoam 2016; 27 (4): 207-14.

8. Cheng XJ, Lin JC, and Tu SP. Etiology and Prevention of Gastric Cancer. Gastrointest Tumors 2016; 3 (1): 25-36.

9. Huang JQ, Sridhar S, Chen Y, Hunt RH. Meta-analysis of the relationship between Helicobacter pylori seropositivity and gastric cancer. Gastroenterology 1998; 114 (6): 1169-79.

10. No authors listed. An international association between Helicobacter pylori infection and gastric cancer. The EUROGAST Study Group. Lancet 1993; 341: 1359-62.

11. Danesh J. Helicobacter pylori infection and gastric cancer: systematic review of the epidemiological studies. Aliment PharmacolTher 1999; 13 (7): 851-6.

12. Helicobacter and Cancer Collaborative Group. Gastric 
cancer and Helicobacter pylori: a combined analysis of 12 case control studies nested within prospective cohorts. Gut 2001; 49 (3): 347-53.

13. Ministerio de Salud. Guía Clínica AUGE Cáncer Gástrico. Santiago, Minsal, 2014.

14. Heise K, Bertran E, Andia ME, Ferreccio C. Incidence and survival of stomach cancer in a high-risk population of Chile. World J Gastroenterol 2009; 15 (15): 1854-62.

15. Dixon MF, Genta RM, Yardley JH, Correa P. Classification and grading of gastritis. The updated Sydney System. International Workshop on the Histopathology of Gastritis, Houston 1994. Am J Surg Pathol 1996; 20 (10): 1161-81.

16. Lee YC, Chiang TH, Chou CK, Tu YK, Liao WC, Wu MS, et al. Association Between Helicobacter pylori Eradication and Gastric Cancer Incidence: A Systematic Review and Meta-analysis. Gastroenterology 2016; 150 (5): 1113-24.

17. Fuccio L, Zagari RM, Eusebi LH, Laterza L, Cennamo V, Ceroni L, et al. Meta-analysis: can Helicobacter pylori eradication treatment reduce the risk for gastric cancer? Ann Intern Med 2009; 151 (2): 121-8.

18. Ford AC, Forman D, Hunt RH, Yuan Y, Moayyedi P. Helicobacter pylori eradication therapy to prevent gastric cancer in healthy asymptomatic infected individuals: systematic review and meta-analysis of randomised controlled trials. BMJ 2014; 348: g3174.

19. Rugge M. Gastric Cancer Risk in Patients with Helicobacter pylori Infection and Following Its Eradication. Gastroenterology Clin North Am 2015; 44 (3): 609-24.

20. Katsinelos P, Tziomalos K, Chatzimavroudis G, Vasiliadis T, Katsinelos T, Pilpilidis I, et al. Eradication Therapy in Helicobacter pylori-Positive Patients with Halitosis: Long-Term Outcome. Med PrincPract 2007; 16 (2): 119-23.

21. Parasakthi K, Peh S, Puthucheary S, Wong N. The rapid urease test in the diagnosis of Helicobatcer pylori infection. Singapore Med J 1994; 35 (2): 161-2.

22. Thillainayagam AV, Arvind AS, Cook RS, Harrison IG, Tabaqchali S, Farthing MJ. Diagnostic efficiency of an ultrarapid endoscopy room test for Helicobacter pylori. Gut 1991; 32 (5): 467-9.

23. Goh KL, Cheah PL, Navaratnam P, Chin SC, Xiao SD. HUITAI Rapid urease test: A new ultra-rapid biopsy urease test for the diagnosis of Helicobacter pylori infection. J Dig Dis 2007; 8 (3): 139-42.

24. Chey WD, Leontiadis GI, Howden CW, Moss SF. ACG Clinical Guideline: Treatment of Helicobacter pylori Infection. Am J Gastroenterol 2017; 112 (2): 212-39.

25. Malfertheiner P, Megzaud F, O’Morain CA, Gisbert JP, Kuipers EJ, Axon AT, et al. Management of Helicobacter pylori infection the Maastricht V/Florence Consensus Report. Gut 2017; 66 (1): 6-30.

26. Abdeljawad K, Wehbeh A, Qayed E. Low Prevalence of Clinically Significant Endoscopic Findings in Outpatients with Dyspepsia. Gastroenterology Res Pract 2017; 2017: 3543681.

27. El-Zimaity H, Serra S, Szentgyorgyi E, Vajpeyi R, Samani A. Gastric biopsies: the gap between evidence-based medicine and daily practice in the management of gastric Helicobacter pylori infection. Can J Gastroenterol 2013; 27 (10): e25-30.

28. Calvet X. Diagnosis of Helicobacter pylori Infection in the Proton Pump Inhibitor Era. Gastroenterol Clin North Am 2015; 44 (3): 507-18.

29. Ricci C, Holton J, Vaira D. Diagnosis of Helicobacter pylori: invasive and noninvasive tests. Best Pract Res ClinGastroenterol 2007; 21: 299-313.

30. Calvet X, Sanfeliu I, Musulen E, Mas P, Dalmau B, Gil $\mathrm{M}$, et al. Evaluation of Helicobacter pylori diagnostic methods in patients with liver cirrhosis. Aliment Pharmacol Ther 2002; 16 (7): 1283-9.

31. Kim DJ, Kim HY, Kim SJ, Hahn TH, Jang MK, Baik $\mathrm{GH}$, et al. Helicobacter pylori Infection and Peptic Ulcer Disease in Patients with Liver Cirrhosis. Korean J Intern Med 2008; 23 (1): 16-21.

32. Xia HH, Phung N, Altiparmak E, Berry A, Matheson M, Talley NJ. Reduction of peptic ulcer disease and Helicobacter pylori infection but increase of reflux esophagitis in Western Sydney between 1990 and 1998. Dig Dis Sci 2001; 46 (12): 2716-23.

33. Kuipers EJ, Thijs JC, Festen HP. The prevalence of Helicobacter pylori in peptic ulcer disease. Aliment Pharmacol Ther 1995; Suppl 2: 59-69.

34. Jang HJ, Choi MH, Shin WG, Kim KH, Chung YW, Kim $\mathrm{KO}$, et al. Has Peptic Ulcer Disease Changed During the Past Ten Years in Korea? A Prospective Multi-center Study. Dig Dis Sci. 2008; 53 (6): 1527-31. 